Port Acadie

Revue interdisciplinaire en études acadiennes

An Interdisciplinary Review in Acadian Studies

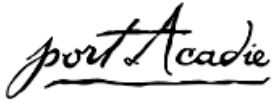

\title{
Catherine Jolicoeur, fille de Marie de l'Assomption
}

\section{Ronald Labelle}

Numéro 24-25-26, automne 2013, printemps-automne 2014

L'Apport des prêtres et des religieux au patrimoine des minorités : parcours comparés Bretagne/Canada français

URI : https://id.erudit.org/iderudit/1019127ar

DOI : https://doi.org/10.7202/1019127ar

Aller au sommaire du numéro

Éditeur(s)

Université Sainte-Anne

ISSN

1498-7651 (imprimé)

1916-7334 (numérique)

Découvrir la revue

\section{Citer cet article}

Labelle, R. (2013). Catherine Jolicoeur, fille de Marie de l'Assomption. Port Acadie, (24-25-26), 96-107. https://doi.org/10.7202/1019127ar

\section{Résumé de l'article}

En tant que spécialiste du légendaire acadien, soeur Catherine Jolicoeur a joué un rôle de premier plan dans le développement de la recherche ethnologique au Canada français à partir de la fin des années 1960 jusqu’aux années 1980, par son travail de terrain, sa participation à des sociétés savantes et son implication dans les grands travaux de classification des légendes. Cette communication présentera le parcours singulier de Catherine Jolicoeur en tant qu'ethnologue rattachée à une congrégation religieuse. 


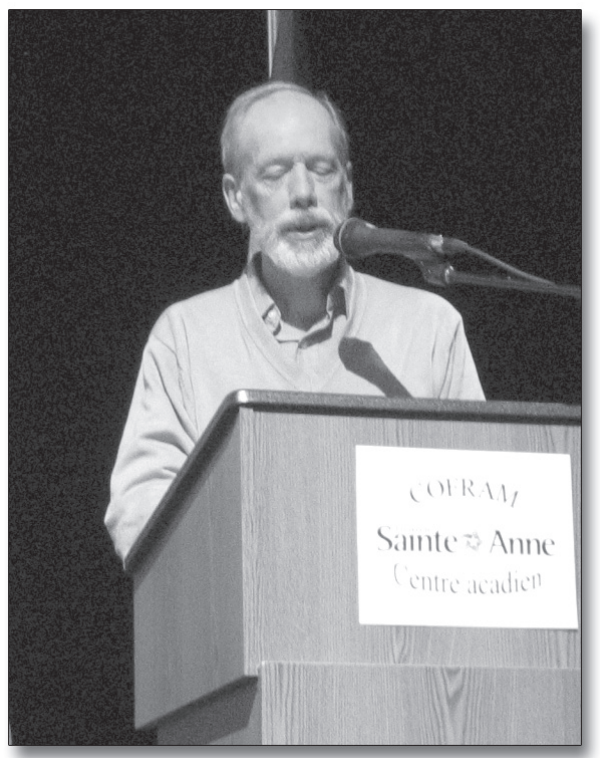

Ronald Labelle 


\title{
Catherine Jolicœur, fille de Marie de l'Assomption
}

\author{
Ronald Labelle \\ Cape Breton University, Sydney, \\ Nouvelle-Écosse
}

\begin{abstract}
Résumé
En tant que spécialiste du légendaire acadien, sœur Catherine Jolicœur a joué un rôle de premier plan dans le développement de la recherche ethnologique au Canada français à partir de la fin des années 1960 jusqu'aux années 1980, par son travail de terrain, sa participation à des sociétés savantes et son implication dans les grands travaux de classification des légendes. Cette communication présentera le parcours singulier de Catherine Jolicœur en tant qu'ethnologue rattachée à une congrégation religieuse.
\end{abstract}

La carrière de sœur Catherine Jolicœur en tant que folkloriste se caractérise par trois mots : ambition, ténacité et persévérance. Sa collecte de témoignages oraux se rapportant aux croyances et légendes est sans doute la plus importante en Amérique française. Pour bien apprécier la productivité de Jolicœur en tant que collectrice, il faut savoir qu'elle a accompli la grande majorité de ses enquêtes pendant une courte période de trois ans, entre 1976 et 1978, alors qu'elle avait déjà atteint l'âge de soixante ans.

Dans un article publié en 1997 intitulé « La Grande CEuvre inachevée de Catherine Jolicœur ${ }^{1}$ ", j'expliquais comment elle s'était empressée de recueillir une quantité extraordinaire de légendes pendant les dernières années de sa carrière, dans le but d'établir un corpus suffisamment grand pour permettre l'élaboration d'une taxonomie des légendes acadiennes. Une telle œuvre aurait pu ensuite servir à des études comparatives internationales. Bien qu'elle n'ait pas achevé son grand projet, elle nous a laissé, en plus de son fonds d'archives, une des meilleures études d'un phénomène légendaire, soit l'ouvrage Le Vaisseau-fantôme : légende étiologique. Cette étude était basée sur une thèse de doctorat préparée en 1963 sous la direction de Luc Lacourcière.

Plusieurs autres religieuses ont rédigé des thèses sur le folklore à l'Université Laval entre les années 1950 et 1970, mais peu ont poursuivi des travaux de collecte une fois leur diplôme obtenu. Dans le cas de Catherine Jolicœur, la thèse n'était que le début d'une carrière de folkloriste qu'elle mena parallèlement à ses activités en tant que religieuse enseignante.

1.

"La Grande Fuvre inachevée de Catherine Jolicœur », dans Pauline Greenhill \& Diane Tye (dir.), Undisciplined Women - Tradition and Culture in Canada, Montréal, McGill-Queen's University Press, 1997, p. 28-38. 
Elle était membre des Filles de Marie de l'Assomption, une congrégation fondée à Campbellton, Nouveau-Brunswick en 1922. Les FMA se vouaient à l'enseignement dans les écoles publiques francophones des provinces Maritimes et de l'est du Québec. Cette vocation correspondait bien aux talents et à la personnalité de Jolicœur, une habile pédagogue qui avait une facilité à communiquer avec les jeunes. Tout au long de sa carrière, elle s'est dévouée à ses trois domaines de prédilection : l'enseignement, la recherche et la vie religieuse.

Catherine Jolicœur, qui possédait un caractère très indépendant et qui avait acquis une réputation internationale pendant les années 1970 en tant qu'ethnologue, n'a jamais mis en question sa vocation religieuse. Cela peut surprendre, car elle œuvrait à une époque où plusieurs de ses consœurs quittaient leur congrégation pour poursuivre une carrière. Dans un texte autobiographique qu'elle rédigea en 1972, elle fit la réflexion suivante qui suggère les tensions qu'elle devait subir au sein de sa congrégation : «Je ne partirai jamais de moi-même. J'attendrai qu'on m'envoie. Et j'attends encore ${ }^{2}$. »

Catherine Jolicœur a suivi un parcours singulier. En 1960, après avoir complété une scolarité de doctorat à l'Université Laval, elle est revenue aux Maritimes où elle a pris un poste d'enseignement dans la petite école primaire de Buttes-Amirault en Nouvelle-Écosse. Sœur Adela Collette, qui travaillait au même endroit à cette époque, lui avait demandé pourquoi elle ne cherchait pas à détenir un poste de professeur d'université au lieu de reprendre l'enseignement dans une école rurale. Catherine Jolicœur lui répondit : " Je me suis donnée au Seigneur et je ne change pas d'idée. Qu'importe que j'enseigne la première année ou que je sois professeure d'université, pourvu que je sois là où le Seigneur me veut ${ }^{3}$. "

J'ai travaillé de près avec sœur Catherine Jolicœur entre 1981 et 1983. Elle m'a confié ses souvenirs à plusieurs reprises pendant mes séjours au Centre universitaire Saint-Louis-Maillet, à Edmundston, ainsi que pendant ses visites aux archives de folklore du Centre d'études acadiennes à Moncton, où elle déposait les résultats de ses recherches. Ceci m’a permis de bien connaître son parcours en tant que collectrice.

Née à Nouvelle en Gaspésie en 1915, elle était la fille d'un cheminot québécois nommé Wilfred Jolicœur et d'une mère de descendance irlandaise, Hélène Parker. Elle passa la plupart de son enfance à Shippagan, Nouveau-Brunswick, puis retourna en Gaspésie avec ses parents en 1927.

2. Cité par sœur Julie d'Amour, f.m.a., dans l'homélie prononcée lors des funérailles de sœur Catherine Jolicœur, le 19 mars 1997. CÉACC, dossier biographique "Catherine Jolicœur ».

3. Adela Collette, f.m.a., En mémoire d'une amie, Catherine Jolicœur, s.l.n.d., p. 7. CÉACC, fonds non classé. 
Elle devint enseignante à l'école du village de Nouvelle alors qu'elle n'avait que 17 ans. Il était courant à l'époque que des jeunes filles soient embauchées comme enseignantes dès la fin de leurs études secondaires. Deux ans plus tard, elle entra comme novice chez les Filles de Marie de l'Assomption à Campbellton, malgré l'opposition farouche de sa mère. En 1937, elle fit sa profession religieuse, prenant le nom de sœur Marie-Sainte-Hélène et elle retourna enseigner, cette fois-ci dans les écoles françaises du nord du Nouveau-Brunswick.

La persévérance de Catherine Jolicœur lui a permis de réussir bien des projets, mais elle pouvait aussi être très têtue, ce qui lui a certainement causé des ennuis. Son parcours révèle que tout au long de sa carrière, elle s'est butée aux autorités dans divers contextes. En salle de classe, par exemple, elle refusait d'imposer la stricte discipline qui était monnaie courante chez les religieuses enseignantes. Elle me confia un jour que, pendant son noviciat, on ne la considérait pas apte à enseigner parce qu'elle perdait du temps en faisant faire de l'exercice physique aux élèves pendant les heures de classe. C'est qu'elle suivait une méthode d'enseignement qui consistait à faire bouger les enfants pour qu'ils soient ensuite bien disposés à travailler. Pendant ses trois années de noviciat, Catherine Jolicœur a donc été rattachée à la maison-mère, où elle était responsable du poulailler, une tâche humiliante pour quelqu'un qui arborait des ambitions d'étudier et d'enseigner. Elle me dit que, pendant ce long purgatoire, elle ne faisait qu'une seule prière, s'adressant directement au Christ, à qui elle disait : "Si c'était pas pour toi, je resterais pas ici ».

Elle reçut enfin la permission de sortir du poulailler pour retourner à l'enseignement et, à partir de 1950, elle s'inscrit à des cours d'été, d'abord au collège de Bathurst et ensuite au collège Saint-Louis d'Edmundston. Puis, en 1956, elle commença ensuite à suivre des cours d'été à l'Université Laval. Loin d'être intéressée par le folklore, elle désirait parfaire ses compétences en littérature. Elle entretint pendant un certain temps une correspondance avec un fils de Paul Claudel alors qu'elle préparait un mémoire de maîtrise intitulé « Notre Dame dans l'œuvre de Claudel 4 ». Dès la réception du diplôme de maîtrise ès lettres en 1959, elle s’inscrit au doctorat à l'Université Laval.

C'est alors que le contact avec le professeur Luc Lacourcière détourna son intérêt vers le folklore. Le discours qu'elle fit lors de la soutenance de sa thèse de doctorat en 1963 décrit de façon éloquente la façon dont ce dernier l'attira vers l'étude des légendes traditionnelles. Cet extrait du dis-

4.

Sœur Marie-Sainte-Hélène, « Notre Dame dans l'œuvre de Claudel », mémoire, M.A., Université Laval, 1958. 
cours de la candidate au doctorat nous permet d'apprécier l'esprit vif que possédait Catherine Jolicœur :

En septembre 1959, nous arrivions à l'Université remplie d'espoirs et d'illusions. [...] on nous conseille de rencontrer le professeur de littérature canadienne. Nous savions que ce professeur était en même temps Directeur des Archives de Folklore et, pour rien au monde, nous aurions voulu entreprendre des recherches dans le domaine folklorique. [...] nous sommes donc retournée à la Direction des thèses. [...] Nous voulions éviter la rencontre fatidique à tout prix. Après avoir attendu deux mois et présenté 18 sujets, nous avons, en désespoir de cause, résolu de franchir le seuil des Archives. [...] le professeur de littérature suggère : "Pourquoi ne feriez-vous pas l'étude d'un conte acadien ? » Voilà ce que nous redoutions le plus ! [...] Puis nous nous sommes mis à la recherche d'un conte acadien intéressant, qui se prêterait sans trop de difficulté ou de tiraillement à une étude scientifique. [...] À la fin de février 1960, nous étions presque découragée et prête à accepter n'importe quel sujet, même le plus futile. C'est à ce moment psychologique que le Directeur des Archives nous demande : "Connaissez-vous la légende du bateau-fantôme ? " Nous nous rappelions vaguement avoir entendu un récit du genre, une vingtaine d'années plus tôt. "Qu'en pensez-vous comme sujet de thèse ? " continue le Directeur. [...] Avec l'enthousiasme d'un condamné à mort gravissant les 13 degrés de l'échafaud, nous avons re-franchi le seuil des Archives. Il y avait exactement 9 versions littéraires au classeur et 16 versions enregistrées dont quelques-unes seulement étaient transcrites. Nous avons donc commencé notre enquête. ${ }^{5}$

Plus loin dans ce même texte, Jolicœur écrit qu'elle a enregistré près de 200 informateurs et témoins des apparitions du vaisseau-fantôme. En tant que religieuse collectrice, elle s'est servie de tous les moyens à sa disposition pour obtenir les renseignements désirés. Sa correspondance nous apprend qu'elle avait demandé l'aide de ses consœurs qui lui ont transmis non seulement leurs propres connaissances mais aussi celles des habitants des villages où elles enseignaient. Certaines ont même enregistré des entrevues sur bande magnétique 6 . Grâce à leur aide, elle a pu distribuer 400 questionnaires sur la légende?

\footnotetext{
5. CÉACC, fonds Catherine-Jolicœur, 63-025, " Soutenance ».

6. CÉACC, fonds Catherine-Jolicœur, 63-021.

7. Catherine Jolicœur, Le Vaisseau-fantôme, légende étiologique, Québec, Presses de l'Université Laval, 1970, p. 1.
} 
Catherine Jolicœur a aussi obtenu la collaboration de plusieurs ethnologues, tant en Amérique du Nord qu'en Europe. L'ethnologue belge Roger Pinon lui a apporté une aide précieuse, obtenant maints renseignements sur le vaisseau-fantôme aux Pays-Bas, qu'il traduisait du néerlandais ${ }^{8}$. La thèse intitulée Le Vaisseau-fantôme : légende étiologique a été publiée en 1970 dans la collection « Les Archives de folklore ». Il s'agit d'un œuvre unique au Canada français : une étude comparative d'une légende à partir de plus de 650 versions orales et écrites. Cette œuvre a même été comparée au Rameau d'or de James Frazer ${ }^{9}$. Catherine Jolicœur a fait preuve d'avant-gardisme, car son étude n'est pas qu'une simple monographie comme on en réalisait à l'époque, mais contient aussi des réflexions sur la psychologie des témoins des apparitions mystérieuses.

Une fois la rédaction de la thèse complétée en 1963, ses activités d'enquête ont été plutôt sporadiques, mais en 1971, Jolicœur a accepté l'offre de travailler auprès de Luc Lacourcière à l'élaboration d'un catalogue analytique des légendes d'Amérique française, financé par la prestigieuse fondation Killam. Pendant les douze dernières années de sa vie active, elle s'est consacrée entièrement à l'étude des traditions orales, sauf pour une année (1974-1975) où elle s'est vue obligée de retourner à l'enseignement dans une école secondaire.

Entre 1971 et 1974, Catherine Jolicœur a préparé, sous la direction de Luc Lacourcière, un ouvrage intitulé "Légendaire d’Amérique française essai de classification ». À l'aide des deux autres participantes au projet, elle a aussi constitué des dossiers sur chaque thème identifié, où étaient rassemblées des fiches bibliographiques, des transcriptions de récits oraux, de la correspondance et des coupures d'articles. Elle a aussi réalisé des études approfondies de certains thèmes, rédigeant, par exemple, un texte intitulé "La légende de la chasse-galerie, ses transformations et sa signification profonde ${ }^{10} »$. Les années 1971 et 1972 ont été fructueuses aussi pour Jolicœur en tant que collectrice. Elle a mené des séries d'entrevues importantes avec deux conteurs du Madawaska au Nouveau-Brunswick, en plus de passer une semaine entière à recueillir des centaines de récits oraux du barde des Îles-de-la-Madeleine, Avila LeBlanc.

Le projet de classification des légendes s'est terminé de façon décevante pour Catherine Jolicœur. Aucun de ses travaux n'a été publié à la fin du projet Killam en 1974 et son essai de classification des légendes, déposé aux Archives de folklore, est aussi demeuré inédit. C'est avec amertume qu'elle a quitté l'Université Laval pour la dernière fois. Elle en voulait évi-

8. Ibid.

9. Luc Lacourcière, «Préface » dans Catherine Jolicœur, Le Vaisseau-fantôme, légende étiologique, op.cit., p. viii.

10. Aful, fonds Luc-Lacourcière, $\mathrm{P}_{178}-\mathrm{C}_{3} / 2,13$. 
demment à Luc Lacourcière pour ne pas avoir mené à terme le catalogue des légendes et elle n'a plus jamais sollicité ses conseils, ni son appui.

Je soupçonne que Lacourcière et Jolicœur ne partageaient pas la même vision de la nature du catalogue, mais les documents qui nous restent de ces deux chercheurs nous éclairent peu sur cette question. Dans une demande de subvention de recherche préparée en 1975, cette dernière proposait de recueillir des légendes acadiennes qui seraient classifiées selon le Motif-Index of Folk Literature d'Aarne-Thompson. Des motifs additionnels y seraient ajoutés, afin de contribuer à l'élaboration d'un nouvel index sémiotique des motifs et des sujets dans la littérature orale populaire ${ }^{11}$. Ce projet était aussi relié au mouvement lancé au début des années 1960 en vue de la réalisation d'un catalogue international de légendes.

Une fois revenue au Nouveau-Brunswick, Catherine Jolicœur a envisagé un travail de cueillette et d'analyse en vue de préparer d'abord un catalogue régional des légendes acadiennes, où des milliers de nouveaux documents oraux s'ajouteraient aux récits déjà répertoriés en archives ${ }^{12}$. Son projet a pris forme grâce à l'appui institutionnel du Centre d'études acadiennes de l'Université de Moncton. C'est le directeur de ce centre, le père Anselme Chiasson, qui lui fournit un lieu de travail. Ayant appris au début 1975 que Jolicœur était revenue enseigner dans le village de Paquetville au Nouveau-Brunswick, ce dernier lui avait immédiatement offert un poste temporaire qui lui fournirait l'encadrement nécessaire pour reprendre son projet de catalogue.

Catherine Jolicœur entreprit sa collecte de légendes à l'été 1975, dès son arrivée au Centre d'études acadiennes (CÉA). Elle enseigna alors un cours d'été sur les traditions orales, où elle donna à ses étudiants la mission de mener des enquêtes orales dans les communautés acadiennes environnantes. Sœur Adela Collette, qui suivait le cours, témoigne que l'enseignante a suscité un grand intérêt chez les étudiants pour la collecte du folklore acadien ${ }^{13}$. Il en résulta des cueillettes importantes, dont les résultats furent déposés aux archives du CÉA.

Jolicœur profita ensuite de ses contacts dans le système scolaire pour visiter vingt écoles du nord du Nouveau-Brunswick et y introduire les enseignants et élèves à la recherche légendaire. Il en résulta une cueillette de 700 textes sur les légendes et croyances. En décembre 1975, elle reçut une subvention du Conseil des arts du Canada ${ }^{14}$ et entreprit immédiatement

11.

12.

13.

14 .
CÉAAC, Archives institutionnelles, fonds B17-228, demande de subvention au Conseil des arts du Canada, juillet 1975.

Ibid.

Entrevue avec sœur Adela Collette, le 2 novembre 2011.

Il s'agit de l'organisme qui subventionnait à l'époque les recherches universitaires en sciences humaines. 
une collecte intensive de légendes dans le sud-est de la province. Chaque entrevue sonore était inventoriée d'une façon détaillée et un numéro d'enregistrement était assigné à chaque passage traitant d'un sujet relié aux croyances traditionnelles. En un an et demi, Catherine Jolicœur parcourut toutes les communautés acadiennes du Sud-Est, recueillant plus de 12000 versions de légendes. Son exploit est d'autant plus remarquable qu'elle ne conduisait pas une voiture, mais dépendait de l'assistance de ses consœurs pour effectuer ses déplacements.

À l'été 1976, alors qu'elle avait à peine commencé sa grande collecte de légendes acadiennes, est arrivée une difficulté qui aurait pu freiner ses activités. Le directeur du CÉA, le père Anselme Chiasson, ayant atteint l'âge obligatoire de la retraite, dut quitter ses fonctions et la bourse de la fondation MacDonald-Stewart qui avait servi à payer un salaire modeste à Jolicœur était épuisée. Le nouveau directeur du centre ne trouva pas le moyen de renouveler son contrat, malgré l'appui dont ses recherches avaient bénéficié de la part du Conseil des arts du Canada.

Deux ans après avoir perdu son poste aux Archives de folklore de l'Université Laval, Catherine Jolicœur s'est donc de nouveau retrouvée sans rattachement institutionnel. Elle demeura une autre année à la résidence des Filles de Marie de l'Assomption à Moncton, continuant son travail d'enquête, d'inventaire et de transcription des légendes. En juin 1977, lorsqu'elle fit au CÉA le dépôt de son importante collection recueillie dans le sud-est de la province, elle remit au nouveau directeur une lettre qui exprimait son mécontentement devant le peu de considération que lui avait accordé l'Université de Moncton ${ }^{15}$.

Ce manque d'appui institutionnel ne ralentit cependant pas son élan. Elle obtint une deuxième subvention du Conseil des arts du Canada qui lui permit d'entreprendre des enquêtes dans le comté de Restigouche au nord de la province, cette fois-ci avec l'assistance d'étudiants enquêteurs. En 1977 et 1978, elle ajouta 5000 enregistrements de légendes et croyances à son inventaire. Catherine Jolicœur prépara alors un plan d'enquête ambitieux qui devait s'étendre à toutes les régions acadiennes des provinces Maritimes. Mais il lui était impossible d'obtenir une subvention pour une recherche s'échelonnant sur plusieurs années sans des assises dans un établissement universitaire.

Heureusement, sa persévérance porta fruit. Elle reçut en 1977 une offre de poste de professeur invitée au Centre universitaire Saint-Louis-Maillet d'Edmundston, dans le nord-ouest du Nouveau-Brunswick. Ce centre, qui serait renommé plus tard "Université de Moncton, campus d'Edmundston », jouissait encore à l'époque d'un certain degré d'autonomie. Jolicœur

15.

CÉACC, fonds Catherine-Jolicœur, 63-021, lettre à Jean Daigle datée le 30 juin 1977. 
y fut accueillie à bras ouverts et c'est là qu'elle œuvra jusqu'à ce que la maladie impose une fin prématurée à sa carrière en 1984 .

Dès son arrivée à Edmundston, elle prépara une importante demande de subvention qui fut approuvée par le Conseil des arts du Canada au printemps 1978, lui permettant d'embaucher une équipe de jeunes chercheurs pour transcrire en entier sa collection de légendes et aussi pour entreprendre des enquêtes dans le Madawaska. Ses trois assistants de recherche ont fait preuve de beaucoup d'ambition et d'enthousiasme, parcourant la région tant du côté canadien qu'américain, car il faut savoir que le Madawaska est une région frontalière.

L'équipe formée de Gordon Boulay, Nicole Dubé et Roch Ringuette a recueilli sous sa direction un total de 292 heures d'enregistrements sonores, comprenant une somme incroyable de renseignements sur les croyances populaires régionales transmis à l'intérieur de milliers de récits légendaires. La classification du matériel a aussi été entreprise dans le cadre du projet de recherche, mais Jolicœur a dû y œuvrer seule, après avoir tenté sans succès d'impliquer un étudiant en ethnologie dans ce travail complexe.

C'est au Madawaska que Catherine Jolicœur s'est vraiment épanouie en tant qu'ethnologue. Elle présenta des conférences sur la taxonomie des légendes acadiennes lors de deux colloques internationaux aux États-Unis en 1978, ainsi qu'au congrès quinquennal de l'International Society for Folk Narrative Research (ISFNR) à Édimbourg en 1979. Elle entra aussi en communication avec des ethnologues américains comme Wayland Hand, de l'Université de Californie, où elle se rendit en 1980 afin d'obtenir des conseils sur l'élaboration d'un catalogue de légendes. Lorsque je rencontrai Wayland Hand en 1984, il me confia qu'il avait essayé de lui apporter le plus d'aide possible, car il reconnaissait qu'elle menait son travail de catalogage dans l'isolement.

Au début 1981, Catherine Jolicœur fit un stage de deux mois à l'University of Southwestern Louisiana à Lafayette, où elle participa à l'organisation des Archives de folklore cadien et créole. Elle profita de son séjour pour mener une série intensive d'enquêtes sur les croyances traditionnelles des Cadiens. Ce stage, financé par le National Endowment for the Humanities, témoigne de la réputation internationale qu'elle avait acquise en tant que spécialiste de la légende.

Au Canada, elle a participé à la même époque à une série de colloques annuels sur la religion populaire coordonnés par Benoît Lacroix, ainsi qu'aux réunions de l'Association canadienne d'ethnologie et de folklore (ACEF), dont elle a assumé la présidence en 1981. Cette même année, elle publia un premier recueil de légendes intitulé Les plus belles légendes 
acadiennes ${ }^{16}$. Ce recueil présentait une sélection de légendes recueillies dans le comté de Westmorland, soit la grande région de Moncton. L'auteur prévoyait publier au moins cinq volumes de légendes du Nouveau-Brunswick, soit un pour chaque région acadienne de la province. Elle ne réussit pas à mener à terme ce projet, bien qu'elle eût deux manuscrits en préparation lorsqu'elle a dû cesser ses travaux. Elle produit quand même une vingtaine d'articles sur les légendes entre 1974 et 1983, dont la plupart parurent dans des revues de sociétés historiques régionales.

En plus de ses activités de recherche, d'enseignement et de publication, Catherine Jolicœur trouva le temps de participer à un projet éducatif intitulé «L'enseignement des quatre savoirs (écouter, parler, lire, écrire) à partir d'un conte populaire ${ }^{17}$ ". Il s'agissait d'un projet pilote où elle visita toutes les écoles primaires du Madawaska canadien, racontant non pas des légendes mais des contes. Après avoir écouté un récit, les enfants s'en inspiraient pour réaliser soit des illustrations ou des projets de rédaction ${ }^{18}$. L'expérience fut ensuite renouvelée dans deux écoles du nord-est de la province. Une enseignante qui accueillit Jolicœur a écrit qu'elle profita de sa visite pour lancer les enfants de la $4^{\mathrm{e}}$ année à la recherche de contes et légendes : " Le lendemain, les élèves sont arrivés avec chacun cinq ou six contes différents. Je me souviens que le premier jour, ils en avaient recueilli cinquante-sept. La joie de Catherine débordait ${ }^{19}$. »

En 1981, alors qu'elle atteignait l'âge de 65 ans, le Centre universitaire Saint-Louis-Maillet lui conféra le titre de professeur associé et, grâce au maintien de son statut de chercheuse, elle a pu solliciter une subvention afin d'étendre ses enquêtes au nord-est du Nouveau-Brunswick. À partir de ce moment-là, elle s'est entièrement dévouée à la recherche et sa passion était telle que ses consœurs se demandaient parfois si elle n'était pas devenue obsédée par les légendes. Partout où elle allait, elle questionnait les gens de son entourage sur leurs connaissances du domaine légendaire, notant sur des bouts de papier tous les faits racontés. Mais rares étaient les religieuses de sa congrégation avec lesquelles elle pouvait s'entretenir de folklore. Dans une correspondance avec une proche collaboratrice datée de septembre 1981, elle écrit : "Note bien que je comprends que les autres n'aient pas mon enthousiasme, car elles ont d'autres goûts et intérêts. Mais je sais que toi, tu comprends ! ${ }^{20} »$

16. Catherine Jolicœur, Les plus belles légendes acadiennes, Montréal, Éditions Stanké, 1981.

17. CÉACC, fonds Catherine-Jolicœur, 63-006.

18. CÉACC, fonds Catherine-Jolicœur, 63-001.

19. Adela Collette, f.m.a., op. cit., p. 17.

20. CÉACC, fonds Catherine-Jolicœur, non classé. 
En 1982, alors qu'elle entreprenait une nouvelle étape dans son projet d'enquête et de classification, Catherine Jolicœur sentait que le temps pressait, car elle commençait à connaître des problèmes de mémoire. Elle tenta d'exercer sa mémoire pour lutter contre la maladie qui se manifestait de plus en plus et elle avoua un jour à une de ses consœurs : " Je me demande ce qui m'attend... Si c'est une maladie grave, je vais lutter jusqu'à la fin. ${ }^{21} »$

La fin de sa carrière fut pénible. Au moment où le Conseil de recherches en sciences humaines du Canada $\left(\mathrm{C}_{R S H}\right)$ lui attribua une nouvelle subvention en 1982, elle ne pouvait plus travailler au même rythme qu'auparavant. Elle tenta quand même de relancer son programme d'enquêtes, mais la collecte menée l'année suivante dans le nord-est de la province a souffert d'un manque de direction, alors que la préparation du grand catalogue des légendes acadiennes était en perte de vitesse. Elle perdit rapidement la bataille contre la maladie d'Alzheimer et devint incapable de poursuivre son projet.

J'ai visité Catherine Jolicœur à quelques reprises pendant l'année universitaire 1983-1984 afin de l'aider à organiser son fonds en vue d'un éventuel dépôt aux archives de folklore du CÉA. Les événements se sont bousculés au milieu de 1984, alors que les membres de sa congrégation se rendaient compte qu'elle était devenue confuse. On l'a alors transportée à l'infirmerie des Filles de Marie de l'Assomption à Campbellton et on a mis un terme au projet financé par le CRSH.

L’année suivante, Jolicœur accepta que les responsables de sa congrégation expédient son fonds documentaire au Centre d'études acadiennes, où il se trouve aujourd'hui, au même endroit que les quelque 900 heures d'enregistrements sonores originaux réalisés par elle-même et ses assistants de recherche. Personne n'a repris le flambeau pour mettre à terme la réalisation d'un catalogue de légendes et le projet de publication d'une anthologie ne s'est pas non plus poursuivi, bien que l'on puisse espérer que ce dernier projet soit éventuellement complété.

L'article que j'ai signé intitulé " La Grande Fuvre inachevée de Catherine Jolicœur » fait partie du collectif Undisciplined Women - Tradition and Culture in Canada ${ }^{22}$. Cet ouvrage paru en 1997 faisait ressortir les difficultés qu'ont connues les femmes à une époque où les institutions du savoir intellectuel étaient dominées par les hommes. Des femmes comme

21. Rita Landry, f.m.a., « Notice biographique de Sœur Catherine Jolicœur », 12 avril 1993.

22. Ronald Labelle, « La Grande Guvre inachevée de Catherine Jolicœur », loc. cit. 
Geneviève Massignon, Helen Creighton et Catherine Jolicœur ont dû montrer beaucoup d'audace et même de bravoure afin d'arriver à leurs fins. Cette dernière a fait preuve d'une persévérance typique des chercheuses de son époque et elle a peut-être été la plus ambitieuse du groupe. Rappelons qu'elle s'était consacrée au catalogage des légendes à une époque où les questions taxonomiques ne connaissaient plus la vogue qu'elles avaient eue dans les années 1950 et 1960. Elle était aussi marginalisée en tant qu'ethnologue travaillant en Acadie à une époque où la recherche ethnologique au Canada français était centrée sur le Québec.

Catherine Jolicœur a surtout été une femme déterminée à préserver la mémoire des anciens. Lorsqu'elle interrogeait ses informateurs, elle se mettait entièrement à leur écoute, ce qui les encourageait à creuser au fond de leur mémoire pour faire jaillir des récits du passé. Selon une de ses proches collaboratrices, elle avait aussi une simplicité désarmante qui favorisait le contact avec les gens ${ }^{23}$. Elle possédait une profonde foi chrétienne dont elle témoignait souvent, mais elle n'avait pas tendance à porter de jugement sur les autres, ce qui favorisait à la fois ses rapports avec ses informateurs et avec ses étudiants. Elle possédait une spiritualité authentique, caractérisée par l'acceptation de son prochain. Cette qualité atténuait, dans une certaine mesure, son caractère têtu.

Il arrive que je trouve un peu déroutante l'écoute des entrevues réalisées par Catherine Jolicœur, parce que je sens qu'elle passait souvent trop vite d'un sujet à un autre, plutôt que de laisser aux informateurs le temps d'approfondir les thèmes abordés. Mais c'était sa façon de procéder. Elle avait l'ambition de recueillir chaque élément de croyance présent dans la mémoire collective des Acadiens. Tout comme son premier maître, Luc Lacourcière, elle était d'abord et avant tout une collectrice. Enfin, cette anecdote racontée par Marie-Élise Ferran, professeur de français au Centre universitaire Saint-Louis-Maillet à la même époque qu'elle, illustre à merveille l'esprit qui animait sœur Catherine Jolicœur :

J'étais son chef de secteur, mais en réalité elle m'embrigadait dans des aventures imprévues. Je me rappelle une collecte de légendes à Rivière-Verte. Après avoir soupé chez une amie, nous avons passé la soirée à écouter tous les conteux de la famille. Pendant qu'elle se berçait en souriant, je devais prendre des notes sur un lot de vieilles enveloppes (car elle avait commencé à recycler le papier bien avant que ça ne fût à la mode). « Et surtout, n’oublie rien... », me disait-elle !24 